\title{
THE SENSOR LOCATION PROBLEM: METHODOLOGICAL APPROACH AND APPLICATION
}

\author{
Evangelos Mitsakis, Evangelia Chrysohoou, Josep Maria Salanova Grau, \\ Panagiotis Iordanopoulos, Georgia Aifadopoulou \\ Centre for Research and Technology Hellas (CERTH), \\ Hellenic Institute of Transport, Thessaloniki, Greece
}

Submitted 30 November 2014; resubmitted 8 April 2015; accepted 30 May 2015; first published online 11 January 2017

\begin{abstract}
The sensor location problem is of particular importance when planning the allocation of limited field equipment intended to be used for advanced traffic management systems and traveller information services. The locations within a network that satisfy specific goals need to be carefully selected, based on predefined goals related to the effective collection of data and the subsequent estimation of traffic related information. The detection of traffic volumes is mainly associated with two purposes, the travel time and the Origin-Destination $(\mathrm{O}-\mathrm{D})$ trip matrix estimation. In this context, this paper presents a quadratic programing model, able to determine the optimal location of tracking sensors. The model is implemented in the urban road network of the city of Thessaloniki (Greece) in which specific number of sensors is installed and utilized for real-time travel time information provision. The proposed methodology models the sensor location problem under the general framework of a set covering problem, which is one of the most popular optimization problems and has been applied in many industrial problems. The results of the case study in Thessaloniki reveal that the proposed model defines the optimal location of the limited number of sensors in such a way that the network, which is created having all sensors as origin or destination of all possible paths, represents to great extent ( $87 \%$ of the traffic flow along the major paths) the traffic volumes of the whole road network of the city.
\end{abstract}

Keywords: sensor location; travel time estimation; BT sensors; advanced traveller information services; point-to-point sensors.

\section{Introduction}

The capabilities for the provision of real-time mobility information and services have significantly increased during the last years, especially due to both new available technologies and high penetration level of smart devices, which have become an indispensable component of the daily traveling.

An emerging technique for the provision of real time travel time as well as for monitoring drivers' behaviour and traffic patterns is the use of point-to-point sensors for tracking individual travellers within a road network. Different kinds of sensors detect various characteristics of traffic flows such as travel time, speed, density, occupancy, volumes. There are mainly four types of sensors in accordance to the flow characteristics that can be obtained: counting sensors (able to count vehicles on a lane(s)), path - ID sensors (measuring the flow volumes of special vehicles having electronic tags and following a predefined path), image sensors (measuring the flow volumes using machine vision) and vehicle - ID sensors (where the vehicles have unique identification characteristics, such as the license plate or RFID detectors).

One technology capable of tracking anonymously users at various locations within a road network is that of Bluetooth (BT) sensors. BT sensors cannot be classified in the conventional ones, since their detections include not only vehicles. However it has been proved by various studies (Haghani et al. 2010; Quayle et al. 2010) that they can be utilized in order to produce travel time of vehicles moving on specific paths.

The market penetration of devices equipped with BT technology has grown significantly during the last years. Nowadays one third of the vehicles have detectable BT on board while $70 \%$ of all new vehicles will have BT connectivity by 2016 (Strategy Analytics 2010). At the same time, there is a significant increase in the sales of the BT enabled devices (mobile phones, e-health

Corresponding author: Josep Maria Salanova Grau

E-mail: jose@certh.gr 
devices, sports and fitness training, e-assistant, wearables, etc.), with more than 7 billion BT enabled devices shipped between 2000 and 2011, while forecasts show that more than 3.5 billion of BT enabled devices will be shipped yearly by 2016 (Powell 2013; Banerjea 2013). Particularly, a comparison between traffic flow values and number of BT detections in Thessaloniki (Greece) in 2012 showed that $20 \%$ of the vehicle traffic volume is detected by the BT sensors.

Within a road network, BT sensors can be placed in selected locations and detect bypassing BT enabled devices, such as navigators, mobile cell phones or other in-vehicle BT devices. Each BT device has a unique Media Access Control (MAC) address, which is stored in the logger of the sensors along with the exact time of the detection. Whenever a BT device is detected by two different sensors at two different locations and time frames the travel time along the route between these two sensors can be measured. It becomes obvious that the location of the BT sensors important and should be carefully examined as a sensor location problem.

In this context, the aim of the current research is to define a methodology for the location of sensors for estimating travel times in an urban area based on a point-to-point tracking system of BT sensors. The methodology is applied to the road network of Thessaloniki, where a network of 35 BT sensors has been installed for the real-time provision of travel times along major routes of the city.

The paper is structured as follows: A literature review is presented in the first section, discussing various existing studies related to the location of traffic sensors in a network as well as to the design of point-to-point sensors networks, especially with the use of BT sensors. The second section presents the proposed methodology followed by its application in the road network of the city of Thessaloniki in the third section. Finally, conclusions and future research directions are presented in the last section of the paper.

\section{Literature Review}

The use of BT sensors has been studied extensively, with positive results regarding their high effectiveness for the estimation of travel times (Ahmed et al. 2008; Sharifi et al. 2011), both when applied to freeways and arterials (Haghani et al. 2010; Quayle et al. 2010; Wasson et al. 2008) and to urban road networks (Mitsakis et al. 2015). The main difference between the freeways and the urban networks is that in the latter the application of such systems presents significant difficulties due to the urban road networks' density and complexity. The determination of the locations where the sensors should be installed is a crucial step, since travel times estimation significantly depends on the sensors' layout. According to literature, there are three main criteria that an optimal sensor location methodology needs to cover: (1) identification of the intersections that are the end points (destinations) of paths with significant variation of travel times within a day, (2) identification of the intersections that serve significant traffic flow volumes, and (3) identification of the intersections through which the drivers can follow alternative routes, in order to avoid congestion. Various methodologies have been developed for determining the optimum sensors' locations, fulfilling the three above mentioned criteria to a significant extent.

Aiming to provide accurate traffic flow estimates Bianco et al. (2001) have emphasized on the importance of the process to identify the total number and the location of sensors. Until then, the only sensor location prerequisite developed was the 'Origin-Destination (O-D) Covering Rule' of Yang et al. (1991), who defined and solved the problem by a two - stage process approach. In the first stage, they solved the sensor location problem in order to determine the minimum number of sensors, while in the second stage they produced an O-D trip matrix. The main idea was that the information derived by the solution of the sensor location problem could improve the accuracy of any applied O-D estimation model.

Gentili and Mirchandani (2005) further investigated the sensor location problem for a class of sensors, which can communicate with the vehicles and collect information regarding their route but also other traffic characteristics. The authors have developed two generic location selection models for active sensors that aim to provide answers to the following two question: (1) which is the quantity and the location of sensors, so as to obtain sufficient information on traffic flow volumes and (2) taking into account that there is already an existing network of sensors, which is the quantity and the location of additional sensors, so as to collect sufficient information regarding traffic flows and volume. The framework of the general set covering problem, which is one of the most popular optimization problems and mostly applied to industrial problems, has been used for all scenarios analysed.

Eisenman et al. (2006) used a simulation-based system for real time network traffic estimation and prediction with the use of dynamic traffic assignment for the analysis of various levels of detections, as well as different sensor locations. They approached the sensor location problem considering the perspective of value of information.

A comprehensive review of the sensor location is provided by Gentili and Mirchandani (2011). They have classified the sensor location - flow estimation problem as an optimization problem according to different evaluation criteria and operational rules.

Sherali et al. (2006) used a quadratic function of multiplication of traffic flow and the coefficient of variation of traffic flow on each link. They proposed a nonlinear mixed integer programming model that can be solved even for large scale networks. Teodorovic et al. (2002) proposed as objective function the weighted average of total number of sensors and the number of $\mathrm{O}-\mathrm{D}$ pairs that are partially covered. Bartin et al. (2007) proposed the minimization of the weighted sum of speed variation of all road segments using a nearest neighbour 
algorithm. Barceló et al. (2012) proposed the use of link detection layout problem with modified sets, including also constrains, with model specific conditions. They also proposed an intersection layout problem. Their model aims to maximize the traffic flow detected over the paths that connect the examined intersections.

Based on review of the related literature, it is apparent that most of the methodologies developed focus on the estimation of $\mathrm{O}-\mathrm{D}$ matrices, and therefore the sensors location aims at maximizing the number of $\mathrm{O}-\mathrm{D}$ pairs and paths covered.

Most authors follow the set covering problem approach. The model proposed in the present paper is based on the dual of the set covering problem. The motivation for using the dual problem approach is to achieve maximum observed traffic volumes, in order to ensure efficiency to the travel time estimation models.

\section{Methodology}

In order to identify the most significant intersections for locating the BT sensors, the following methodological steps have been followed (Fig. 1).

\subsection{Identification of the Road Links Comprising the Network of the Study Area}

The first step entails the selection of the links that comprise the road network of the study area, which is a subset of all physical links. This procedure aims to exclude links with limited capacity, which may not be included in travellers' route choices. The network is selected to include only significant links, which are connected to major intersections.

\subsection{Identification of Major Paths and Candidate Locations}

The candidate BT sensor locations are at the intersections of the major paths, since by locating the sensors at intersections the total number of detected vehicles is larger in comparison to locating the sensors between intersections, considering that the detections will include the vehicles coming from and heading to all the legs of the intersection. In order to analyse the characteristics of the selected intersections (candidate BT sensor locations) and in order to define comparison mechanisms, the most significant (major) paths are defined, based on the traffic flow volume.

\subsection{Optimization Model for Solving the Sensor Location Problem}

The objective function and the constraints of the proposed optimization model are defined as follows: the overall objective is to identify the set of intersections (sensor locations) that maximizes the observed (daily) traffic flow volumes. Thus, the decision variable $x$ is binary and defined as follows:

$$
x_{i}= \begin{cases}1, & \text { when intersection } i \text { is selected } \\ 0, & \text { otherwise. }\end{cases}
$$

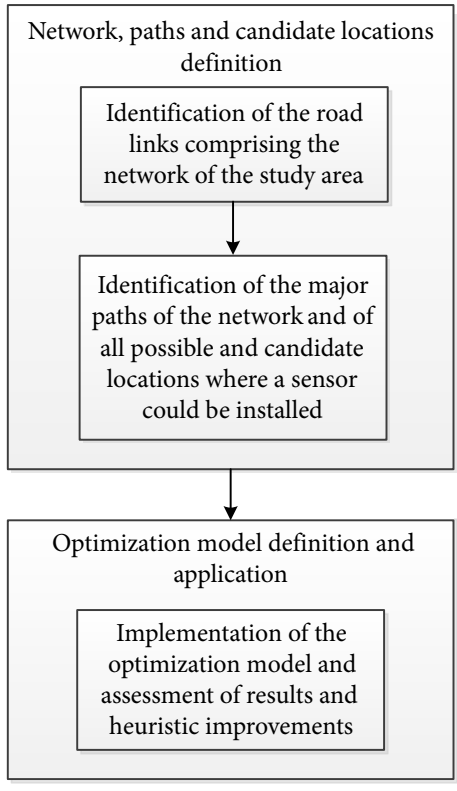

Fig. 1. Methodological flowchart

The objective function of the optimization model is defined as:

$$
\max \sum_{i=1}^{n} h_{i} \cdot x_{i}
$$

with $h_{i}$ corresponding to the average hourly traffic flow volume that can be detected at the intersection $i$.

In order to calculate the total hourly traffic flow volume for each intersection (Fig. 2.), entering and exiting traffic flow volumes of all corresponding intersection legs are calculated for every hour of the day based on the following formula:

$$
h_{i}=\frac{1}{2} \cdot \sum_{j=1}^{A_{i}} a_{i j},
$$

where: $A_{i}$ is the number of legs of intersection $i$; $a_{i j}$ is the traffic flow volume on leg $j$ of intersection $i$.

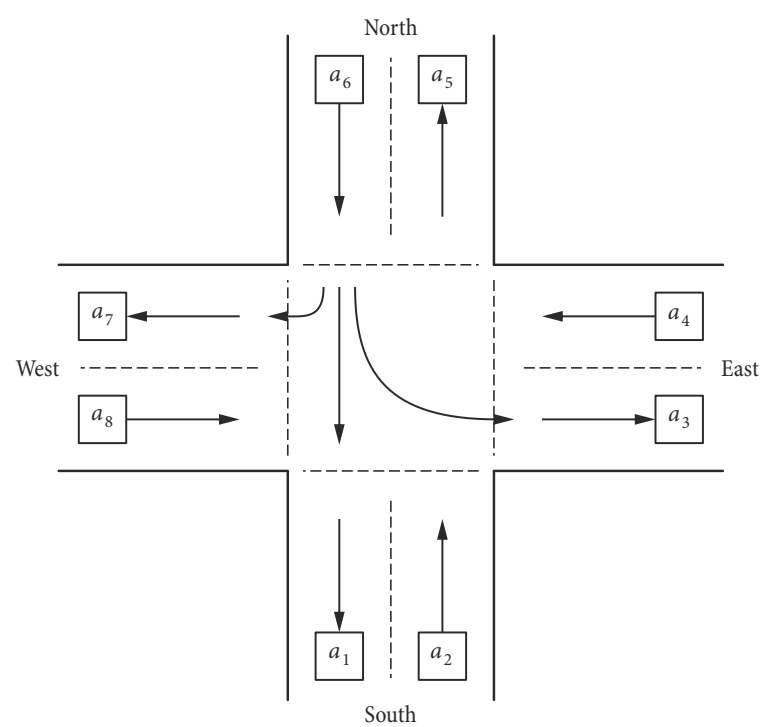

Fig. 2. Intersection layout 
There are the following constraints:

- the existing network of BT sensors;

- the total number of sensors to be installed;

- the corresponding 'neighbour' constraint defining the minimum distance between two sensors.

From the coordinates of each intersection, a distance matrix is calculated, expressing the Euclidean distances between each pair of intersections. Intersections located at a specific distance, lower than a predefined limit, which depends on the size of the area and the number of sensors, are defined as neighbouring intersections. The neighbouring indicator matrix is used for ensuring that neighbouring intersections will not be selected. However, the intersections where existing sensors are located are excluded from this procedure.

Parameter $n_{i j}$ of the neighbouring indicator matrix is equal to 1 , when the distance among two intersections is less than $1.5 \mathrm{~km}$ and 0 otherwise. Constraint 1 expressing this rule is specified as follows:

$$
\sum_{i=1}^{n} \sum_{j=1}^{n} x_{i} \cdot n_{i j} \cdot x_{j} \leq 0
$$

Constraint 2 expresses the limitation of the available set of sensors:

$$
\sum_{i=1}^{n} x_{i} \leq Q
$$

where: $Q$ is the number of available sensors.

An additional constraint is included, guarantying that the location of the existing sensors will be included in the result:

$$
\begin{aligned}
& \sum_{i=1}^{n} x_{i} \cdot y_{i}=D \\
& \sum_{i=1}^{n} y_{i}=D
\end{aligned}
$$

where: $D$ is the number of existing sensors; $y_{i}$ indicates that the intersection $i$ belongs to the existing network of sensors.

Since Constraint 1 is quadratic, the optimization model is a Quadratic Programming Model.

\section{Case Study}

A detection network comprised of 10 BT sensors has been operational in the central part of Thessaloniki (Mitsakis et al. 2015, 2013; Morfoulaki et al. 2011) since 2011. This network provides data for estimating and monitoring travel times along 22. Besides the estimation of travel times for informing travellers through an advanced traveller information system, estimates for travel times are also being used for the calibration of a traffic assignment model that has been developed for traffic state estimation and prediction at network level (Stamos et al. 2011). Recently, 25 additional BT sensors have been installed based on the application of the methodology presented in Section 2. The steps and results are presented in the subchapters below.

\subsection{Identification of the Road Links Comprising the Network of the Study Area}

The links that have been selected based on the above criteria are presented in Fig. 3. The entire physical road network of the study area in Thessaloniki is comprised of 137852 links, while the selected network includes 3073 links. This subnetwork is comprised of the main streets of the urban area of Thessaloniki in terms of capacities and traffic volumes and represents $78 \%$ of the total traffic volumes of the city's road network. Moreover, the subnetwork was selected so as to be a fully connected network.

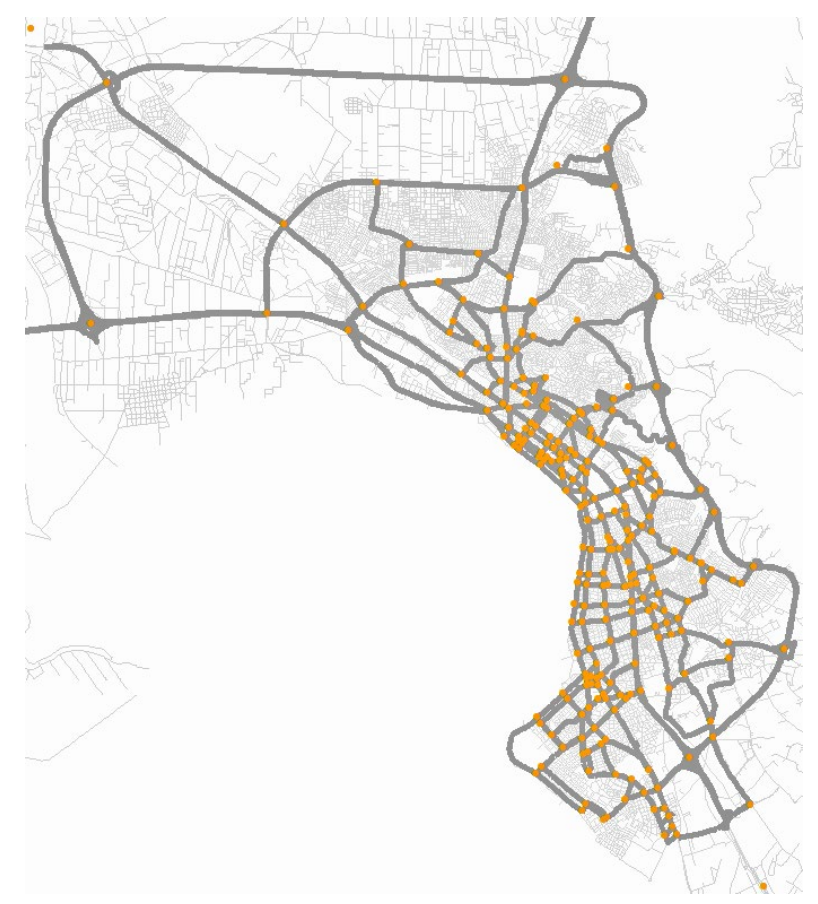

Fig. 3. Intersections and links of the study area of Thessaloniki

\subsection{Identification of Major Paths and Candidate Locations}

The major paths have been selected based on a grid approach, including the main vertical and horizontal axes of the study area. Moreover, additional paths serving short but equally significant trips that are generated mainly in the city centre have been also defined. Intersections that are included in these paths accounts for almost $80 \%$ of the total traffic volumes of the city.

The horizontal paths cover all trips from the main East-to-West and West-to-East 'gates' of the city. It is should be highlighted that the horizontal paths, which fulfil the condition of crossing from East-to-West and vice versa, are limited in number. Initial conclusions based on their main characteristics are the following:

- the observed daily traffic volumes along the peripheral ring road are on average 110000 vehicles with an average speed ranging between 70 $100 \mathrm{~km} / \mathrm{h}$, resulting to travel times of approximately $20 \mathrm{~min}$ for traveling along the entire ring road; 
- the average speed along the main roads passing through the city centre ranges between $40-50 \mathrm{~km} / \mathrm{h}$, the duration of trips is $25 \mathrm{~min}$ on average and the observed traffic volumes are on average 45000 vehicles per day;

- when roads of lower importance (based on the network's hierarchy) are also taken into account, the average speed drops to $25-30 \mathrm{~km} / \mathrm{h}$ and traffic volumes are on average 25000 vehicles per day.

Vertical paths connect the main horizontal axes and thus produce several alternatives, serving the entire city. Some important observations are the following:

- vertical paths located at the Eastern part have an average length of 2-4 km and 7-9 min of travel time;

- vertical paths located at the Central area have an average length of 3-4 km and $10 \mathrm{~min}$ of travel time;

- vertical paths located at the Western part have an average length of 4-6 km and 10 min of travel time.

The intersections that constitute the nodes of the defined network were all candidate locations, where the BT sensors could be located. The total number of intersections of the selected network is 252 .

\subsection{Results}

The optimal solution of the model, taking into account the neighbour constraint as well as the 10 sensors already installed (Fig. 4), is 172741 (vehicles).

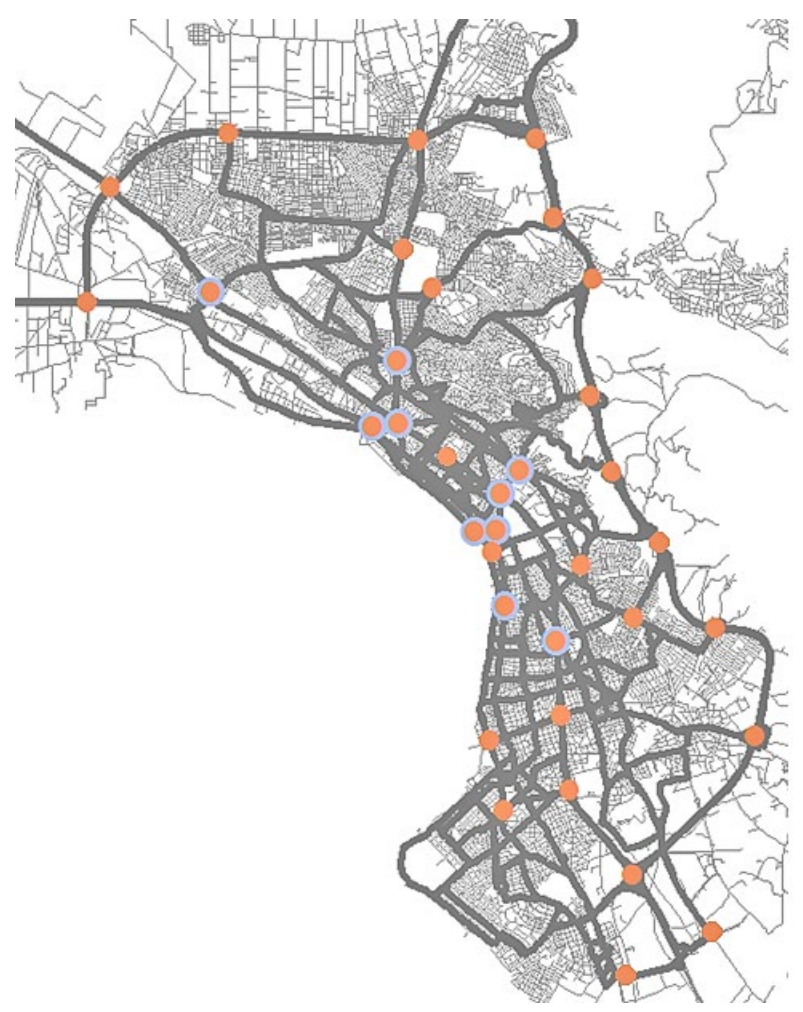

Fig. 4. Optimal sensor locations based on the quadratic model

\subsection{Sensitivity Analysis}

In order to evaluate the robustness of the results, a sensitivity analysis has been performed, aiming at validating the selection of the sensors location based on the results of the optimization model applied to various scenarios.

The model has been applied to hourly traffic flows and to two representative time intervals within the day. The first scenario (A) was obtained by using the average traffic volumes of a typical day, while an average volume for the morning peak period (from 7:00 am to 8:00 pm) was used for the second scenario (B).

The variability of the traffic flow volumes during the day is presented in the box plot of Fig. 5, from which it can be concluded that the variability of traffic flow is significant for several paths.

Fig. 6 shows the box plots of the two scenarios, from which it can be concluded that the traffic flow variability is significantly lower from 7:00 am to 8:00 pm (scenario B).

The optimization model was used for solving the 24 scenarios of the hourly traffic flows, as well as the two scenarios containing average volume values. A total of 38 different locations were obtained from the different solutions. The frequency of appearance of each location is presented in Fig. 7.

A subset with the same 15 locations appeared in the results of all the scenarios, while a larger subset, containing 25 locations appeared in more than $95 \%$ of the solutions.

In order to further validate the results of the applied model, two performance measurements were examined. The first one is the percentage of the coverage of vertical and horizontal major paths, while the second one refers to the percentage of the coverage of daily traffic along these paths. It has been found that $100 \%$ of the horizontal and $75 \%$ of the vertical paths are covered by the sensor locations derived by the proposed model. In total, these account for $87 \%$ of the traffic flow along the major paths.

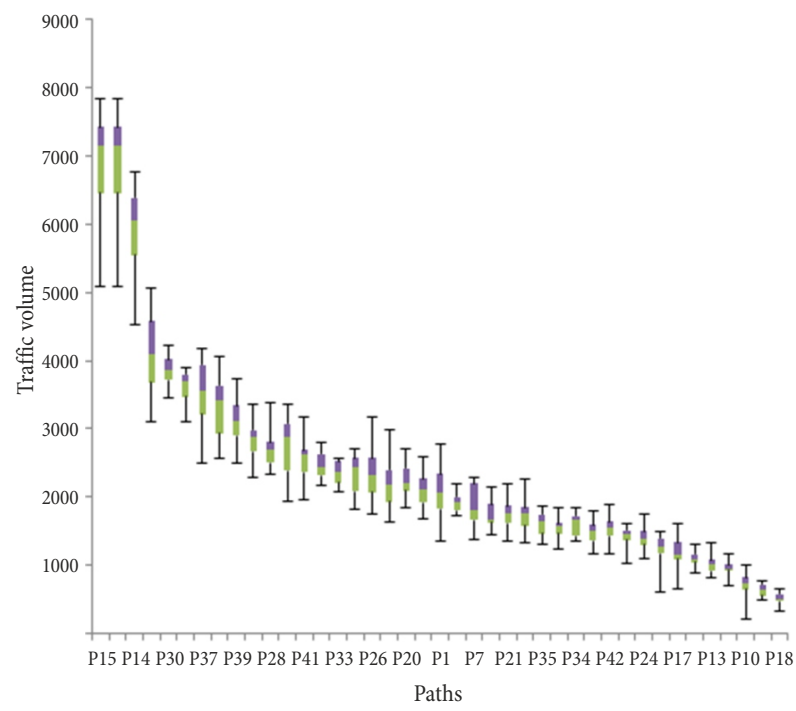

Fig. 5. Variability of traffic volume during the day 

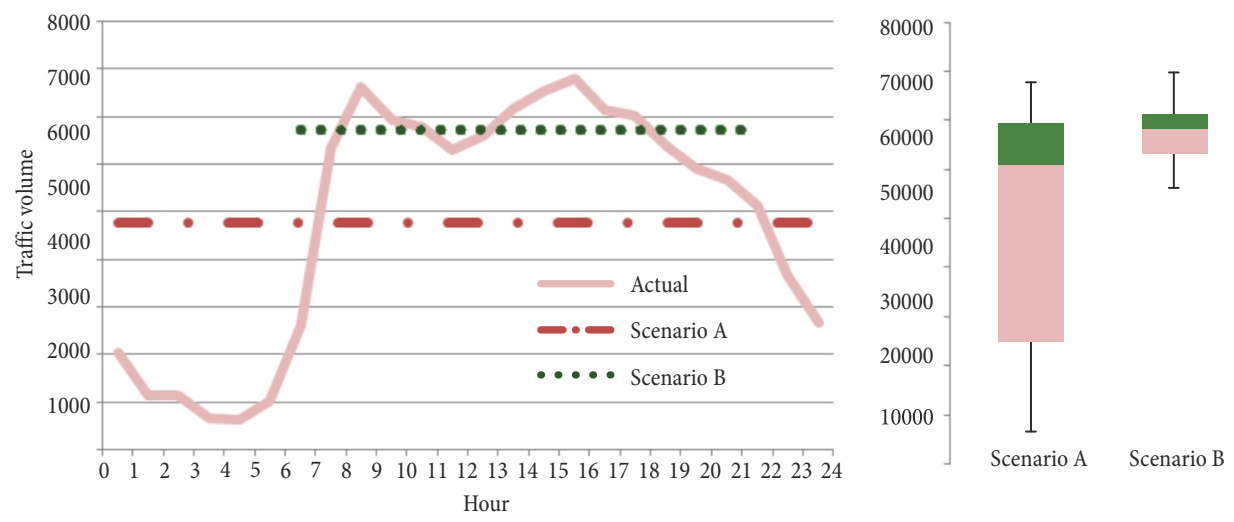

Fig. 6. Variability of traffic volumes

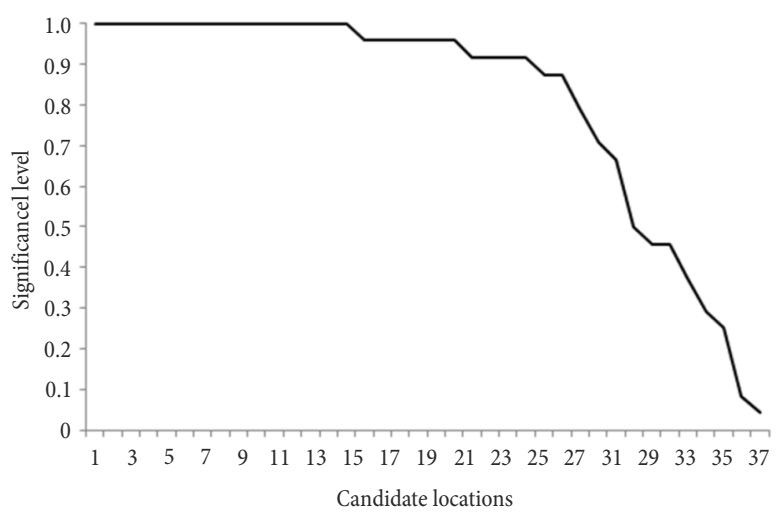

Fig. 7. Frequency of appearance of candidate locations in solutions of the scenarios

\section{Conclusions}

Determining the location of a limited number of field equipment in a road network to be subsequently used for advanced traffic management and traveller information systems is a complex task. The sensors' locations within a network should be selected based on specific goals such as maximizing the traffic flow monitored by the sensors and accounting for variability of flows.

In this paper, a methodology for the optimal location of BT sensors in an urban road network has been proposed, applied and assessed. The methodological framework takes into account the main goals predefined by the specificities of each road network. In the previous sections, all necessary methodological steps have been presented together with the quadratic programming model developed for identifying the optimal locations, taking into account already existing sensors.

The implementation of the proposed modelling approach in the case of Thessaloniki reveals that the result of this approach can effectively meet the three criteria set in the literature. More specifically, the location of the sensors deriving from the methodology are at intersections that serve significant traffic flows of the study area and through which the drivers can follow alternative routes, in order to avoid congested routes. Moreover, their location within the road network can 'shape' a network of paths with significant within-day variations of travel times and serve significant numbers of traffic flow volumes.
Future steps should include research along three main directions. The first one is on the application of alternative solution methods, including the linearization of the product of the variables and the problem formulation as an integer linear programming model. Other approaches such as heuristics and metaheuristics could also be applied. The second direction of further research could focus on the evaluation of the results derived from the location of the sensors with a view to improve other tools and systems that aim to provide real time information to travellers. A third direction of further research includes the optimal allocation of different types of detection sensors, co-existing in the same network (e.g. BT detectors and loop detectors.

\section{Acknowledgements}

This work was supported in part by the South East Europe Transnational Cooperation Programme of the European Regional Development Fund, under the SEE-ITS project.

\section{References}

Ahmed, H.; El-Darieby, M.; Abdulhai, B.; Morgan, Y. 2008. Bluetooth- and Wi-Fi-based mesh network platform for traffic monitoring, in TRB 87th Annual Meeting Compendium of Papers DVD, 13-17 January 2008, Washington, DC, US, 1-11.

Banerjea, R. 2013. The Bluetooth Boom - Connecting Everything. 21 p. Available from Internet: http://www.comsocscv. org/docs/20130710-csr-banerjea.pdf

Barceló, J.; Gilliéron, F.; Linares, M.; Serch, O.; Montero, L. 2012. Exploring link covering and node covering formulations of detection layout problem, Transportation Research Record: Journal of the Transportation Research Board 2308: 17-26. http://doi.org/10.3141/2308-03

Bartin, B.; Ozbay, K.; Iyigun, C. 2007. Clustering-based methodology for determining optimal roadway configuration of detectors for travel time estimation, Transportation Research Record: Journal of the Transportation Research Board 2000: 98-115. http://doi.org/10.3141/2000-12

Bianco, L.; Confessore, G.; Reverberi, P. 2001. A network based model for traffic sensor location with implications on O/D matrix estimates, Transportation Science 35(1): 50-60. http://doi.org/10.1287/trsc.35.1.50.10140 
Eisenman, S.; Fei, X.; Zhou, X.; Mahmassani, H. 2006. Number and location of sensors for real-time network traffic estimation and prediction: sensitivity analysis, Transportation Research Record: Journal of the Transportation Research Board 1964: 253-259. http://doi.org/10.3141/1964-28

Gentili, M.; Mirchandani, P. 2011. Survey of models to locate sensors to estimate traffic flows, Transportation Research Record: Journal of the Transportation Research Board 2243: 108-116. http://doi.org/10.3141/2243-13

Gentili, M.; Mirchandani, P. 2005. Locating active sensors on traffic networks, Annals of Operations Research 136(1): 229-257. http://doi.org/10.1007/s10479-005-2047-z

Haghani, A.; Hamedi, M.; Sadabadi, K.; Young, S.; Tarnoff, P. 2010. Data collection of freeway travel time ground truth with bluetooth sensors, Transportation Research Record: Journal of the Transportation Research Board 2160: 60-68. http://doi.org/10.3141/2160-07

Mitsakis, E.; Salanova Grau, J. M.; Chrysochoou, E.; Aifadopoulou, G. 2015. A robust method for real time estimation of travel times for dense urban road networks using pointto-point detectors, Transport 30(3): 264-272. http://doi.org/10.3846/16484142.2015.1078845

Mitsakis, E.; Stamos, I.; Salanova Grau, J. M.; Chrysochoou, E.; Iordanopoulos, P.; Aifadopoulou, G. 2013. Urban mobility indicators for Thessaloniki, Journal of Traffic and Logistics Engineering 1(2): 148-152. http://doi.org/10.12720/jtle.1.2.148-152

Morfoulaki, M.; Mitsakis, E.; Chrysostomou, K.; Stamos, I. 2011. The contribution of urban mobility management to trip planning and the environmental upgrade of urban areas, Procedia - Social and Behavioral Sciences 20: 162-170. http://doi.org/10.1016/j.sbspro.2011.08.021

Powell, M. 2013. The Bluetooth Boom - Connecting Everything. 13 p. Available from Internet: http://www.comsocscv.org/ docs/20130710-btsig-powell.pdf

Quayle, S.; Koonce, P; DePencier, D.; Bullock, D. 2010. Arterial performance measures with media access control readers: Portland, Oregon, pilot study, Transportation Research Record: Journal of the Transportation Research Board 2192: 185-193. http://doi.org/10.3141/2192-18

Sharifi, E.; Hamedi, M.; Haghani, A.; Sadrsadat, H. 2011. Analysis of vehicle detection rate for Bluetooth traffic sensors: a case study in Maryland and Delaware, in Proceedings of the 18th World Congress on Intelligent Transport Systems, 16-20 October 2011, Orlando, FL, 1-12.

Sherali, H. D.; Desai, J.; Rakha, H. 2006. A discrete optimization approach for locating automatic vehicle identification readers for the provision of roadway travel times, Transportation Research Part B: Methodological 40(10): 857-871. http://doi.org/10.1016/j.trb.2005.11.003

Stamos, I. M.; Salanova Grau, J. M.; Mitsakis, E. A.; Ayfadopoulou, G. V. 2011. Large scale dynamic traffic assignment model for real-time traveler information services, in ITS and Smart Cities 2014, 19-22 November 2014, Patras, Greece, 1-11. http://doi.org/10.13140/RG.2.1.3307.9528

Strategy Analytics. 2010. Global Automotive Vehicle-Device Connectivity Forecast 2008-2016. Available from Internet: http://www.strategyanalytics.com

Teodorovic, D.; Van Aerde, M.; Zhu, F.; Dion, F. 2002. Genetic algorithms approach to the problem of the automated vehicle identification equipment locations, Journal of Advanced Transportation 36(1): 1-21. http://doi.org/10.1002/atr.5670360102
Wasson, J. S.; Sturdevant, J. R.; Bullock, D. M. 2008. Real-time travel time estimates using media access control address matching, ITE Journal 78(6): 20-23.

Yang, H.; Iida, Y.; Sasaki, T. 1991. An analysis of the reliability of an origin-destination trip matrix estimated from traffic counts, Transportation Research Part B: Methodological 25(5): 351-363.

http://doi.org/10.1016/0191-2615(91)90028-H 